\title{
Acute kidney injury in the patient with cancer
}

\author{
Mitchell H. Rosner ${ }^{1 \mathbb{D}}$, Mark A. Perazella ${ }^{2}$ (i) \\ 'Division of Nephrology, University of Virginia Health System, Charlottesville, VA, USA \\ 2Department of Internal Medicine, Section of Nephrology, Yale University School of Medicine, New Haven, CT, USA
}

Dramatic advances in the care of patients with cancer have led to significant improvement in outcomes and survival. However, renal manifestations of the underlying cancer as well as the effects of anti-neoplastic therapies leave patients with significant morbidity and chronic kidney disease risks. The most common renal manifestations associated with cancer include acute kidney injury (AKI) in the setting of multiple myeloma, tumor lysis syndrome, post-hematopoietic stem cell therapy, and AKI associated with chemotherapy. Knowledge of specific risk factors, modification of risk and careful attention to rapid AKI diagnosis are critical for improving outcomes.

Keywords: Acute kidney injury, Chemotherapy, Neoplasms, Oncology

\section{Introduction}

Patients with cancer are at high risk for infections, sepsis, tumor lysis syndrome (TLS), drug-associated toxicities, and other comorbidities that significantly increase the likelihood of developing acute kidney injury (AKI) $[1,2]$. The development of AKI in these patients represents a significant event that increases mortality and morbidity and can limit the effectiveness and use of chemotherapeutic regimens $[1,2]$. Unfortunately, no effective therapies for AKI exist, making prevention critically important. Prevention of AKI rests on the recognition of patient- and cancer-specific risk factors that can be targeted for intervention to lower the likelihood of AKI.

Received April 15, 2019; Revised May 5, 2019;

Accepted May 13, 2019

Edited by Soo Wan Kim, Chonnam National University, Gwangju, Korea

Correspondence: Mitchell H. Rosner

Division of Nephrology, University of Virginia Health System, Charlottesville, VA 22908, USA. E-mail:mhrgr@virginia.edu

Copyright (C) 2019 by The Korean Society of Nephrology

(a) This is an open-access article distributed under the terms of the Creative Commons Attribution Non-Commercial License (http://creativecommons. org/licenses/by-nc-nd/4.0/), which permits unrestricted non-commercial use, distribution, and reproduction in any medium, provided the original work is properly cited.

\section{Epidemiology}

Two recent studies described an overall one-year incidence of AKI in cancer patients between $11 \%$ and $20 \%$, with higher risks in patients with hematological cancers $[3,4]$. Most recently, a study from China that surveyed over 7 million patients demonstrated an incidence of AKI (defined as at least a 50\% increase in baseline serum creatinine) at 14 to $20 \%$ depending upon the hospital type (community vs. academic, respectively) [5]. Some studies have noted much higher rates of AKI (60\%), but are biased with a larger number of critically ill patients with hematological malignancies $[6,7]$. Studies support that the highest incidences of AKI occur with renal cell cancer, liver cancer, multiple myeloma, leukemia, and post-hematopoietic stem cell transplantation (HSCT) $[3,4]$. In a large Danish cohort of patients with cancer and AKI, 5\% required renal replacement therapy (RRT) within one year of AKI onset [4]. However, studies in higher risk, critically ill populations have reported the need for RRT in $8 \%$ to $60 \%$ of patients depending on the severity of AKI and underlying comorbidities [8]. A recent study investigated the incidence of AKI in 163,071 patients receiving systemic treatment (presumably a smaller subset of patients with malignancy) [9], and identified 10,880 patients who experienced AKI. The rate of AKI was 27 per 
1,000 person-years, with an overall cumulative incidence of 9.3\%. Malignancies with the highest 5-year AKI incidence were myeloma (26.0\%), bladder cancer (19.0\%), and leukemia (15.4\%). Advanced cancer stage, chronic kidney disease (CKD), and diabetes were associated with an increased risk of AKI (adjusted hazard ratios [aHR] = $1.41,95 \%$ confidence interval $[\mathrm{CI}]=1.28$ to 1.54 ; $\mathrm{aHR}=$ $1.80,95 \% \mathrm{CI}=1.67$ to 1.93 ; and $\mathrm{aHR}=1.43,95 \% \mathrm{CI}=1.37$ to 1.50 , respectively). Interestingly, the annual incidence of AKI increased from 18 to 52 per 1,000 person-years between 2007 and 2014.

Risk factors associated with the development of AKI in cancer patients are both cancer-specific and patientspecific (Table 1). Knowledge of these risk factors is imperative for both prevention and early recognition of AKI. Clearly, hematological malignancies, older age and the presence of underlying CKD represent the greatest baseline risk factors that interact with specific types of chemotherapy to determine the overall AKI risk. This effect is modified by the occurrence of complications such as sepsis, which may significantly increase AKI risk.

AKI in cancer patients has numerous deleterious consequences, including increased mortality (especially for those with higher AKI stages, post-HSCT or requiring RRT), increased the length of hospital stay, and in one study, a lower rate of complete cancer remission $[7,10-$ 15]. In patients undergoing myeloablative conditioning regimens as part of stem cell transplants, those patients with AKI had worse overall survival and progression-

Table 1. Risk factors and etiologies of acute kidney injury in critically ill patients with cancer

\begin{tabular}{ll}
\hline \multicolumn{1}{c}{ Patient-specific risk factors } & \multicolumn{1}{c}{ Cancer-related risk factors } \\
\hline Age $>65 \mathrm{yr}$ & Neutropenia and resulting sepsis \\
Underlying CKD & Post-nephrectomy for RCC \\
Diabetes mellitus & Hematological cancers \\
Potential nephrotoxin medications & Urinary tract obstruction \\
(NSAIDs, ACEi, ARBs) & Post-HSCT \\
Comorbid conditions & Thrombotic microangiopathy \\
(such as cirrhosis, heart & Tumor lysis syndrome \\
failure, nephrotic syndrome) & Hypercalcemia \\
& Paraneoplastic glomerular diseases \\
& Chemotherapy toxicities \\
\hline
\end{tabular}

Individual risk for acute kidney injury is due to a combination of host and cancerrelated factors.

$\mathrm{ACEi}$, angiotensin converting enzyme inhibitor; ARB, angiotensin receptor blocker; CKD, chronic kidney disease; HSCT, hematopoietic stem cell transplant; NSAIDs, non-steroidal anti-inflammatory drugs; RCC, renal cell cancer. free survival as well as increased risk for CKD development [16]. As another example, Libório and colleagues [15] found that mortality was $13.6 \%$ in those without AKI, and progressively increased with higher RIFLE (Risk, Injury, Failure, Loss, End stage renal disease) stage AKI (Risk, 49\%; Injury, 62.3\%; Failure, 86.8\%). Salahudeen et al [3] also demonstrated a decrease in survival in cancer patients with AKI. Using modified RIFLE criteria, $12 \%$ of patients admitted to the hospital had AKI, with rates in the Risk, Injury, and Failure categories of $68 \%, 21 \%$, and $11 \%$, respectively. Dialysis was required in $4 \%$ of patients. In a multivariate model, the odds ratio (OR) for developing AKI was significantly higher for patients with diabetes (OR, 1.89; 95\% CI, 1.51-2.36), receiving chemotherapy (OR, 1.61; 95\% CI, 1.26-2.05), receiving intravenous contrast (OR, 4.55; 95\% CI, 3.51-5.89), and antibiotics (OR, 1.52; 95\% CI, 1.15-2.02). In patients with AKI, length of stay (100\%), cost (106\%), and odds for mortality (4.7-fold) were significantly greater. In addition, AKI in patients with newly diagnosed hematological malignancies was associated with a lower 6 -month complete remission rate (39.4\% in patients with AKI vs. $68.3 \%$ in patients without AKI) and $14.6 \%$ of patients with AKI received suboptimal chemotherapy [10]. Thus, the development of AKI can negatively impact current or future chemotherapeutic regimens, as well as potentially increase the toxicity or alter the pharmacokinetics and pharmacodynamics of these drugs. Furthermore, AKI would exclude patients from potentially beneficial clinical trials.

The long-term consequences of AKI in the patient with cancer are highly variable and it is likely that overall severity of illness, age, and functional status contributes significantly to prognosis in these patients. Thus, while some studies report poor 30-day survival in patients with AKI and cancer, other studies have not found a difference [17]. While the impact of AKI on long-term kidney function has been rarely reported in this subset of patients, this outcome also appears to be variable. One study reported that $82 \%$ of critically ill cancer patients with AKI completely recovered kidney function, while partial recovery was observed in $12 \%$, and chronic RRT was required in only $6 \%$ of patients [11]. Other studies have reported long-term dialysis dependence in 12.9 to $23 \%$ of patients with hematological malignancies who develop dialysis-requiring AKI $[6,14]$. These data highlight the importance of careful and individualized decision mak- 
ing in patients with AKI, as some patients will have good outcomes.

\section{AKI in hematological malignancies}

Patients with hematological malignancies (including leukemia, lymphoma, and multiple myeloma) are at the highest risk for AKI development in most case series. AKI may be due to the direct effects of the malignancy, such as with the development of light chain cast nephropathy in patients with multiple myeloma, or may be due to downstream effects of therapy, such as with sepsis associated with immunosuppression and neutropenia [18]. Many AKI etiologies in the setting of hematological malignancies are rare, and a kidney biopsy may be required for accurate diagnosis (Table 2). A thorough diagnostic process should be undertaken in patients with AKI and hematological cancers, including urinalysis with quantification of urine protein excretion (albumin, protein electrophoresis, and total protein), free light chain (FLC) quantification, serum chemistries, complete blood counts, and renal ultrasound.

A particularly insidious cause of AKI is tumor cell infiltration of the kidneys, which is most common with lymphoma and leukemia. In these cases, renal ultrasound findings of bilaterally enlarged kidneys should prompt consideration of leukemic or lymphomatous infiltration. AKI likely results from tumor cells that cause tubular compression and disruption of the renal microcircula-

Table 2. Etiologies of acute kidney injury in patients with hematological malignancies

\begin{tabular}{cc}
$\begin{array}{c}\text { General non-specific } \\
\text { etiologies }\end{array}$ & $\begin{array}{c}\text { Tumor-related } \\
\text { etiologies }\end{array}$ \\
\hline $\begin{array}{c}\text { Volume depletion } \\
\text { secondary to nausea, } \\
\text { vomiting, diarrhea }\end{array}$ & $\begin{array}{c}\text { Tumor (leukemia or lymphoma) } \\
\text { infiltration of the kidney } \\
\text { Sepsis } \\
\text { lodinated contrast }\end{array}$ \\
Obstructive nephropathy due to \\
retroperitoneal lymphadenopathy \\
(lymphoma) \\
Lysozymuria in chronic myelomonocytic \\
leukemia and acute monocytic leukemia \\
Disseminated intravascular coagulation \\
Tumor lysis syndrome \\
Hypercalcemia \\
Glomerular diseases \\
Myeloma-specific etiologies such as \\
cast nephropathy \\
Chemotherapy-related nephrotoxicity
\end{tabular}

tion [19-21]. AKI in these cases is often rapidly reversible with appropriate and effective chemotherapy. However, these patients are at risk for TLS and should be managed aggressively to prevent this complication (see below).

The findings of proteinuria, microscopic hematuria, or red blood cell casts should prompt consideration of cancer-related glomerulonephritis such as membranoproliferative glomerulonephritis or amyloidosis [2]. In these cases, a kidney biopsy is critical in defining diagnosis and prognosis, along with outlining appropriate treatment. While relatively rare, malignancy-related glomerulonephritis may be the first manifestation of cancer. Thus, age-appropriate cancer screening should be performed in patients with this new diagnosis [22].

Lysozymuria (the presence of large amounts of the enzyme lysozyme in the urine) is an uncommon disorder seen in patients with acute promyelocytic, monocytic leukemia, or chronic myelomonocytic leukemia where malignant cells produce large amounts of lysozyme [23]. Lysozyme is filtered at the glomerulus and taken up by proximal tubular cells, leading to cellular damage and AKI. Urine protein electrophoresis (UPEP) can demonstrate large quantities of lysozyme in these cases. In addition, electrolyte abnormalities such as refractory hypokalemia may also be present.

Patients with multiple myeloma represent an important subclass of patients with hematological malignancies that are prone to develop AKI. The etiologies of AKI in these patients are protean and diverse (Table 3 ). AKI is quite common, complicating the course of myeloma in up to $20 \%$ to $50 \%$ of cases $[24,25]$. The most common cause of AKI in multiple myeloma is cast nephropathy. The updated 2014 criteria of the International Myeloma Working Group consider AKI by light chain cast nephropathy as a myeloma defining event [26]. Light chain cast nephropathy can rarely be associated with other hematological cancers such as Waldenstrom macroglobulinemia,

Table 3. Etiologies of acute kidney injury in patients with multiple myeloma

\begin{tabular}{lc}
\hline \multicolumn{1}{c}{ Paraprotein-related } & Metabolic disturbances \\
\hline Light chain cast nephropathy & Hypercalcemia secondary \\
Light chain related proximal tubular & to bone involvement \\
injury with or without Fanconi syndrome & Hyperuricemia with large \\
Light chain deposition disease & tumor burden \\
$\begin{array}{l}\text { Amyloidosis (more common with } \\
\text { lambda light chains) }\end{array}$ \\
\hline
\end{tabular}


chronic lymphocytic leukemia, or lymphoma [27].

Light chain cast nephropathy develops when FLCs, which are freely filtered by the glomerulus, bind to Tamm-Horsfall protein (uromodulin) in the thick ascending limb of the loop of Henle to form insoluble casts that obstruct the tubular lumen and lead to local inflammation $[28,29]$. There are common binding sites on kappa and lambda light chains which interact in a noncovalent manner with carbohydrate moieties on TammHorsfall proteins [30]. These interactions and the formation of obstructing casts are promoted in the setting of reduced tubular flow rates as well as when the concentrations of urinary electrolytes (sodium and chloride) are higher (such as with diuretic use) [31]. There is also a direct relationship between the risk of cast nephropathy and the serum concentration of FLCs [32]. Histological examination reveals diffuse interstitial inflammation, which may be triggered by leakage of light chains into the kidney interstitium. This leads to activation of multiple pro-inflammatory pathways, and on-going inflammation may progress to irreversible kidney injury, highlighting the importance of early and aggressive therapy [33].

The diagnosis of cast nephropathy centers on measurement of serum FLCs with quantitative measurement of kappa and lambda FLCs as well as serum protein electrophoresis (SPEP) and UPEP [34,35]. Serum FLCs and SPEP identify the presence of pathogenic FLC, and UPEP helps to distinguish paraprotein-related glomerular diseases characterized by albuminuria from cast nephropathy, where proteinuria is largely non-albumin FLCs [36]. A study from the Mayo Clinic demonstrated that in patients with light chain cast nephropathy, urine albumin excretion was less than $25 \%$ of total urine protein excretion, with a median of $7 \%$ [37]. Higher levels of urine albumin excretion should prompt consideration of kidney biopsy. In addition, the International Myeloma Working Group recommends a kidney biopsy to determine alternative causes of AKI if serum FLC levels are less than $500 \mathrm{mg} / \mathrm{L}$ (or $50 \mathrm{mg} / \mathrm{dL}$ ) [26].

Treatment of cast nephropathy has evolved considerably in the last decade and centers on provision of adequate hydration to augment tubular flow and treat preexisting volume depletion ("flushing out the tubular casts"), along with chemotherapy to rapidly reduce FLC levels. Obviously, any potentially nephrotoxic medications should be stopped and avoided. Effective chemo- therapy regimens include proteasome inhibitors such as bortezomib along with other agents such as thalidomide, corticosteroids, vincristine, and adriamycin in various combinations [38-41]. These regimens have been associated with high rates of improvement in renal function as well as significantly improved survival [38-41]. Importantly, bortezomib has the added benefit of acting quickly to improve glomerular filtration rate (GFR) with a median time of response at 1.34 months [42]. The addition of the alkylating agent bendamustine to a regimen of prednisone and bortezomib has also increased renal response rates to greater than $80 \%$, with the majority of the response occurring within 6 weeks [43].

Given that a rapid reduction in the serum concentration of FLCs is critical for improving kidney function, there is continued interest in the use of extracorporeal therapies to rapidly remove FLC while more definitive chemotherapy is being implemented. Thus, the use of therapeutic plasma exchange (TPE) or high-cutoff hemodialysis (using large pore dialysis membranes to facilitate FLC removal) remains of great interest and also of great controversy. In terms of plasmapheresis, the randomized controlled trials have been small (the largest included 97 patients) and inconclusive [44]. Most recently, the use of high flux dialyzers with greater capacity for light chain removal has been studied, and two recent trials have been reported. In the European trial of FLC removal by extended hemodialysis (EuLITE), there was no benefit noted with a high flux dialyzer over conventional therapy [45]. However, high flux dialysis was found to be beneficial in the MYRE trial, which enrolled patients with biopsy-proven cast nephropathy [46]. In this trial, dialysis independence increased to $56.5 \%$ in the high flux dialysis arm, compared to $35.4 \%$ in the conventional arm $(P=0.04)$. Thus, it remains unclear if high-cutoff hemodialysis provides benefit with conflicting evidence in the two trials [47].

\section{Hematopoietic stem cell transplantation}

HSCT is an important and possibly curative treatment for cancer patients, especially those with hematological malignancies. However, AKI may complicate HSCT as a result of conditioning chemotherapy, radiation exposure, sepsis, sinusoidal obstruction syndrome (SOS), thrombotic microangiopathy (TMA), graft-versus-host disease 
(GVHD), or nephrotoxic medications $[48,49]$. The incidence of HSCT-associated AKI ranges from 15\% to $73 \%$ depending on whether an allogenic or autologous transplant is performed and high-dose or reduced intensity chemotherapeutic conditioning regimens are employed [48]. Myeloablative regimens and allogenic HSCT are associated with a higher rate of AKI [50-52]. The need for RRT develops in $\sim 5 \%$ of patients but approaches $30 \%$ in high-risk patients [52-55]. When AKI occurs in this setting, it is associated with an increase in mortality rate, especially when it occurs early in the post-HSCT course, prior to engraftment [56]. Post-HSCT AKI is challenging to treat, as it commonly occurs in patients who are severely immunocompromised and may be manifesting other complications such as GVHD, sepsis, and other critical illnesses. Of great importance is the link between the occurrence of AKI post-HSCT and the eventual development of CKD. Hingorani [57] reported that the cumulative incidence of CKD varies between $7 \%$ and $48 \%$ and develops between 6 months and 10 years after HSCT. Risk factors for CKD include prior AKI, acute and chronic GVHD, older age at HSCT, a decrease in the GFR at baseline, hypertension, the use of calcineurin inhibitors, and exposure to total-body irradiation.

Development of HSCT-associated AKI occurs due to a number of insults, some of which are specific to this clinical scenario and others that are more general, such as sepsis. Liver injury during HSCT (especially during the conditioning regimen) may lead to hepatic SOS, which is an independent risk factor for AKI [58]. The mean incidence of SOS is $13.7 \%$ but is significantly decreasing with newer regimens [58]. Hepatic sinusoidal obstruction occurs due to sinusoidal endothelial cell and hepatocyte damage induced by cytoreductive regimens [58-60]. Hepatic SOS is characterized by painful hepatomegaly, jaundice, oliguria, and ascites and mimics hepatorenal syndrome. Hypervolemia in these cases is usually diuretic resistant, and spontaneous recovery is rare. AKI adversely affects survival, with mortality approaching $80 \%$ in those who require RRT. Prevention and treatment include infusions of prostaglandin-E, pentoxifylline, and low-dose heparin [60-62]. Early administration of defibrotide, an antithrombotic and fibrinolytic agent, appears to beneficial, and if instituted early, may lead to improvements in GFR [63,64].

In HSCT-associated TMA, patients may develop AKI or eventually CKD [65,66]. The lesion is characterized by endothelial swelling and damage, with fibrin thrombi within capillary loops and arterioles $[67,68]$. The conditioning regimens for HSCT can induce renal endothelial injury with subsequent TMA. GVHD may also contribute to TMA due to direct endothelial cell injury as well as from calcineurin inhibitor use $[69,70]$. Treatment includes discontinuing/reducing calcineurin inhibitor dose, TPE, and defibrotide [71-75]. Reported response rates for TPE range between $27 \%$ and $80 \%$ [ $76-81$ ], and $64 \%$ for TPE plus cyclosporine withdrawal [78]. Rituximab may be beneficial for TMA post-HSCT, but more data is needed for this strategy [71,82-86].

Acute GVHD is another significant risk factor for the development of AKI in HSCT recipients [87]. GVHD causes AKI through cytokine-mediated renal inflammation or from cyclosporine exposure. Other AKI etiologies in patients with GVHD include vomiting and diarrhea, which can promote prerenal AKI, as well as viral reactivation (cytomegalovirus). In addition to supportive measures, treatment of GVHD includes use of prednisone, antithymocyte globulin, sirolimus, and mycophenolate mofetil [88].

\section{Tumor lysis syndrome}

TLS is a medical emergency and a common cause of cancer-induced AKI [89]. Risk factors for the development of TLS include highly chemosensitive malignancies such as lymphomas and leukemias, large tumor burden, effective cytolytic chemotherapeutic agents, elevated lactate dehydrogenase levels ( $>1,500 \mathrm{IU})$, and underlying kidney disease $[90,91]$. The most common malignancies associated with TLS include non-Hodgkin's lymphoma, acute myeloid leukemia, acute lymphocytic leukemia, and various solid tumors [92]. The in-hospital mortality associated with TLS can approach $21 \%$, and nearly $70 \%$ of patients experience a severe complication such as sepsis, dialysis, acute respiratory failure, mechanical ventilation, cardiac arrest, or seizures [92]. The median hospital length of stay for patients with TLS is 10 days, but this increases to 21 days if dialysis is required [92].

TLS is characterized by the release of cellular contents from tumor cells that are either spontaneously dying or killed by chemotherapy. These cellular contents can lead to hyperuricemia, hyperkalemia, hyperphosphatemia, 
and hypocalcemia. While there are no universally accepted diagnostic criteria for TLS, the Cairo-Bishop definition of both laboratory and clinical criteria are commonly utilized [93]. Laboratory criteria include the following: hyperuricemia ( $>8 \mathrm{mg} / \mathrm{dL}$ or a $25 \%$ increase from baseline), hyperkalemia ( $>6 \mathrm{mmol} / \mathrm{L}$ or $25 \%$ increase from baseline), hyperphosphatemia ( $>4.5 \mathrm{mg} / \mathrm{dL}$ or $25 \%$ increase from baseline) and hypocalcemia $(<7 \mathrm{mg} / \mathrm{dL}$ or a $25 \%$ decrease from baseline). Laboratory criteria for TLS require the presence of 2 or more of these abnormalities occurring 3 days before or 7 days after therapy. Clinical criteria include serum creatinine elevation $>1.5$ times the upper limit of normal, cardiac arrhythmias, sudden death, and seizures.

AKI in TLS occurs due to a combination of cytokine release with inflammatory tubular injury, acute uric acid/ xanthine nephropathy, and acute nephrocalcinosis due to an elevated calcium-phosphate product [2]. Uric acid, calcium-phosphate, and/or xanthine crystals can lead to tubular obstruction and tubulointerstitial inflammation. Hyperuricemia can also contribute to AKI through renal vasoconstriction, reactive oxygen species generation, and inflammatory cytokine release $[2,94]$.

Diagnosis of AKI attributable to TLS requires an increase in serum creatinine along with fulfillment of laboratory criteria for TLS [89]. AKI typically develops 24 hours or later after initiation of chemotherapy. The clinical presentation depends on the combination and severity of biochemical abnormalities. For instance, if the potassium level rises high enough, patients may experience muscle weakness or cardiac arrhythmias.

Occasionally, cases of spontaneous TLS are seen. Patients with hyperuricemia (uric acid $\geq 8 \mathrm{mg} / \mathrm{dL}$ ) in the presence of suspected malignancy with elevated lactate dehydrogenase ( $>2 \times$ upper limit of normal), acute oliguric or anuric kidney injury despite adequate volume resuscitation without evidence of post-obstructive cause, and urinary uric acid to creatinine ratio greater than 1.0 should be considered to have spontaneous TLS until proven otherwise. Diagnostically, uric acid crystals free or within casts may be seen on urine sediment examination.

Prophylaxis against TLS is recommended for all patients with hematological malignancies undergoing chemotherapy. Prophylaxis is also recommended for all high and moderate risk patients such as those with large tumor burdens, reduced GFR, and highly chemosensitive tumors. However, the exact regimen for prophylaxis should be tailored to the clinical circumstances and includes a combination of decreasing uric acid levels, ensuring adequate hydration and tubular urine flow rate, and management of abnormal electrolyte levels. Prevention and treatment of TLS complications include administration of intravenous fluids ( $3 \mathrm{~L} /$ day) and xanthine oxidase inhibition (allopurinol or febuxostat) in highrisk patients prior to chemotherapy [90,91,94]. Hydration decreases extracellular uric acid, phosphorus and potassium concentrations, enhances renal blood flow and maintains GFR, which aids in maintenance of normal electrolyte levels. Ideally, intravenous hydration is started 24 to 48 hours pre-therapy.

It is also important to monitor for volume overload and use diuretics only when indicated.

Allopurinol is an isomer of hypoxanthine and inhibits the enzyme xanthine oxidase, thereby reducing uric acid synthesis. Allopurinol will increase plasma concentrations of the uric acid precursors hypoxanthine and xanthine, which can form crystals and deposit in the kidney in the presence of an alkaline urine, leading to xanthine nephropathy.

Side effects of allopurinol include fever, rash, eosinophilia, systemic hypersensitivity reactions, StevensJohnson syndrome, hepatitis, acute interstitial nephritis (AIN), and bone marrow suppression. Allopurinol should be started 2 to 3 days prior to therapy and continued for 10 to 14 days. It is important to realize that xanthine oxidase inhibitors will prevent de-novo elevations in uric acid levels but will not lower pre-existing high uric acid levels. Febuxostat is a non-purine analogue xanthine oxidase inhibitor and is useful in patients that are intolerant to allopurinol. One recent trial demonstrated that febuxostat was superior in lowering uric acid levels but there was no difference in clinical outcomes vs. allopurinol [95]. Febuxostat dosing should be $40 \mathrm{mg}$ daily in patients with severe kidney function impairment.

In patients with hyperuricemia present at diagnosis as well as underlying AKI or CKD, recombinant urate oxidase (rasburicase) may be employed to correct hyperuricemia. Rasburicase catalyzes uric acid formation to soluble allantoin, which is rapidly excreted by the kidney. The drug has a rapid onset (within 4 hours) and leads to dramatic falls in serum uric acid levels. Rasburicase is 
indicated for a single course of treatment for the management of plasma uric acid levels in pediatric and adult patients with leukemia, lymphoma, and solid tumor malignancies who are receiving anti-cancer therapy expected to result in tumor lysis and elevation of plasma uric acid. Rasburicase generates hydrogen peroxide in the conversion of uric acid to allantoin and thus is contraindicated in patients with glucose-6-phosphate dehydrogenase deficiency [96-98].

The need for hemodialysis to treat TLS has likely declined since the advent of rasburicase. However, hemodialysis remains a highly effective therapy that can be used to gain control of electrolyte and acid-base issues, especially in the presence of oliguric AKI. Continuous renal replacement therapies can be utilized in the treatment of TLS and have the advantage of avoiding "rebound" metabolic disturbances. If a continuous renal replacement is utilized, higher clearance levels (at least $30-40 \mathrm{~mL} / \mathrm{kg}$ / hour) should be targeted.

\section{Hypercalcemia-induced AKI}

Hypercalcemia occurs in approximately $20 \%$ to $30 \%$ of all malignancies (especially, multiple myeloma and squamous cell carcinomas) and is a common cause of AKI $[99,100]$. In some scenarios, such as with multiple myeloma, the presence of hypercalcemia may potentiate other AKI etiologies. Hypercalcemia promotes direct afferent arteriolar vasoconstriction and also leads to volume depletion from excessive renal sodium and water loss [101]. Hypercalcemia causes sodium wasting at the loop of Henle by activating the calcium-sensing receptor, and also leads to renal water losses by blocking arginine vasopressin activity in the distal nephrons [2]. Rarely, severe hypercalcemia may cause AKI via intratubular calcium-phosphate deposition (nephrocalcinosis), especially if the calcium-phosphate concentration product is higher than $70 \mathrm{mg}^{2} / \mathrm{dL}^{2}$. Of note, nephrocalcinosis-associated AKI may not improve with treatment of hypercalcemia; depending upon the duration of hypercalcemia, patients may be left with significant CKD. This highlights the need for rapid lowering of serum calcium levels.

Patients often present clinically with weakness, dizziness, nausea, and polyuria along with hypotension, dry mucous membranes, and flat neck veins. Treatment of AKI centers around intravascular volume resuscitation to improve renal perfusion and GFR. This enhances calcium excretion and corrects hypercalcemia [99]. In addition to aggressive intravenous fluids with normal saline (without diuretics unless hypervolemia is present), low dose pamidronate (typically $60 \mathrm{mg}$ or lower) infused over 4 hours will also lower serum calcium over a period of several days and has a longer lasting effect to prevent recurrent hypercalcemia $[99,102]$. Denosumab, a humanized monoclonal antibody against receptor activator of nuclear factor- $\kappa \mathrm{B}$ (RANK) ligand, is also effective and does not require dose adjustment for GFR [103,104]. Rarely, dialysis may be required in the setting of severe hypercalcemia and AKI $[99,105]$. This is especially true if the calciumphosphate concentration product is very high.

\section{Drug-induced AKI}

Drug-induced AKI occurs primarily from acute tubular injury (ATI), AIN, and a variety of glomerular and vascular injuries [106-110]. Given the explosion of novel agents to treat cancer, it is imperative that nephrologists stay up to date with the toxicities of these drugs. Broadly, chemotherapy-associated AKI can be separated into 3 drug classes: 1) conventional chemotherapy, 2) targeted therapies, and 3) novel immunotherapies [106-114].

\section{Conventional chemotherapy}

Conventional chemotherapeutic agents may injure all compartments of the kidney (tubules, interstitium, vasculature, and glomerulus) and thus lead to various forms of AKI. TMA is a particularly aggressive form of TMA that may lead to irreversible AKI and the need for dialysis. TMA complicates therapy with gemcitabine, mitomycin C, and cisplatin [106,107,109,114-117]. Drug-induced endothelial injury with release of von Willibrand factor multimers and plasminogen activator inhibitor, as well as exposure of a denuded endothelial surface to fibrin and platelets, facilitates the process within the renal microvasculature [106,107,109,115-117]. While drug discontinuation is required, therapy with several modalities has been disappointing. TPE is generally ineffective but may be attempted, and eculizumab as well as rituximab have only been rarely reported in case series [115-117].

Glomerular injury, specifically podocyte injury with the histological subtypes of focal segmental glomerulo- 
sclerosis (FSGS) or minimal change disease, has been described with pamidronate and, rarely, zoledronate $[118,119]$. In most of these cases, the onset of kidney dysfunction occurs over a more extended period of time and is associated with significant proteinuria.

Several drugs cause AKI due to ATI, which is the most common lesion associated with AKI from conventional chemotherapy $[106,107,109]$. The platinum agents (cisand carboplatin), ifosfamide, pemetrexed, zoledronate, and other agents damage the tubular epithelium via direct cellular toxicity, activation of apoptosis, generation of reactive oxygen species and oxidative stress, and mitochondrial injury $[106,107,109]$. Although drug discontinuation and supportive care improve kidney function, CKD may be a complication. In general, the risk of resulting CKD increases with both the severity and duration of AKI.

Methotrexate is associated with crystalline-induced AKI $[106,107,109,120]$. In this setting, intratubular crystal precipitation with obstructive and inflammatory interstitial injury promotes AKI. While intravenous fluids and urinary alkalinization are used for prevention and treatment, hemodialysis and glucarbidase may be required for severe toxicity $[121,122]$. Renal clearance of methotrexate is of critical importance. In the setting of AKI, toxic levels of methotrexate may accumulate, leading to severe bone marrow toxicity. Thus, AKI associated with methotrexate is a medical emergency and clinicians should monitor GFR closely in these situations and consider early use of hemodialysis or glucarbidase if methotrexate levels rise. Finally, a number of chemotherapeutic agents including carboplatin, ifosfamide, and adriamycin may cause AKI due to interstitial nephritis $[106,107,109,123]$.

\section{Targeted agents}

This class of drugs consists of agents designed to target specific gene mutations that categorize particular cancers, thereby inhibiting oncogenic signaling cascades associated with tumor growth [108,111,124-127]. These drugs have been very successful in effectively treating cancer, but unfortunately are also associated with AKI, proteinuria, hypertension, and electrolyte disturbances $[108,111,124-127]$. In many cases, this is due to the fact that the pathways involved in oncogenesis may have overlapping functions in the kidney.
Anti-angiogenesis drugs (such as bevacizumab, axitinitib, sorafenib, and sunitinib) that target vascular endothelial growth factor (VEGF) cause AKI primarily via renal TMA, although FSGS and AIN have also been observed along with severe hypertension $[108,124,125]$. Doserelated AKI is noted with serine/threonine kinase BRAF inhibitors (vemurafenib and dabrafenib) $[111,126]$. Acute tubulointerstitial injury presumably occurs due to inhibition of the mitogen-activated protein kinase pathway; however, limited histological data is available $[111,126]$. Drug discontinuation is associated with AKI reversal in a majority of cases. Crizotinib, an anaplastic lymphoma kinase inhibitor, causes AKI via tubulointerstitial injury that is partially reversible with drug discontinuation $[112,127]$. This is an evolving area and clinicians should remain suspicious of drug toxicity in any patient with unexplained AKI, and kidney biopsy should be considered.

\section{Novel immunotherapies}

Immunotherapies are an important addition to cancer therapy [128-133]. Older agents such as interferon (IFN) and high-dose interleukin (IL)-2 are well-known causes of AKI $[128,129]$. IFN-associated AKI often presents clinically with high-grade proteinuria from FSGS or minimal change disease [129]. Direct binding of IFN to podocyte receptors and alteration of normal cellular proliferation may promote podocyte injury, although the cytokines IL-6 and -13 may also play a role [128]. Drug discontinuation (+/- steroids) may reverse AKI and proteinuria with minimal change disease, but is less effective in FSGS.

Immune checkpoint inhibitors (ipilimumab, nivolum$a b$, and pembrolizumab) enhance tumor killing by preventing dendritic cells and tumor antigen ligand binding to cytotoxic T-lymphocyte-associated protein (CTLA)4 and programmed death (PD-1) receptors, respectively $[113,114,130]$. This activates and further increases T-cell killing of tumor cells. Unfortunately, loss of tolerance to self and perhaps exogenous medications that have a predisposition to lead to an immune reaction, leads to AIN and a variety of glomerular lesions $[113,114,130]$. Drug discontinuation plus steroids is generally effective in reversing AKI, especially if treatment is started early, but a significant number of patients are left with CKD. However, it is critically important to realize that AKI in the setting of immune checkpoint inhibitors may be associated 
with tubular or glomerular injury and thus, kidney biopsy should be considered.

Chimeric antigen receptor (CAR) T-cells are host cells that are harvested and engineered to express receptors that recognize and bind tumor antigens [131]. T-cells directly target and destroy cancer cells. However, this process promotes macrophage activation and cytokine release syndrome, which can result in capillary leak and prerenal AKI $[132,133]$. TLS may also develop and risks AKI $[132,133]$. Prevention and treatment of AKI include pretreatment chemotherapy to reduce the tumor burden and steroids [132,133]. In the setting of severe cytokine release syndrome, an IL-6 receptor blocker and/or steroids may reduce adverse effects [132,133].

\section{Summary}

Dramatic advances in the care of patients with cancer have occurred in a short period of time and have led to demonstrable increases in longevity. However, a consequence of these advanced therapies has been the occurrence of AKI in many forms. Rapid recognition of AKI as well as appropriate therapy is critical to sustain the gains in outcomes associated with novel chemotherapeutics. Increasingly, AKI etiologies in patients with cancer have become more complex and multi-factorial (for example, chemotherapy-induced AKI exacerbated by the development of sepsis in a patient with neutropenia). Clinicians should think broadly about the many possible AKI etiologies and, in uncertain cases, kidney biopsy should be considered. Lastly, when possible, there should be a focus on AKI prevention, as AKI has the potential to significantly worsen outcomes and limit available therapies for cancer treatment.

\section{Conflicts of interest}

All authors have no conflicts of interest to declare.

\section{Authors' contributions}

Mitchell H. Rosner and Mark A. Perazella contributed equallly to the preparation of the manuscript.

\section{References}

[1] Lameire N, Vanholder R, Van Biesen W, Benoit D. Acute kidney injury in critically ill cancer patients: an update. Crit Care 2016;20:209.

[2] Rosner MH, Perazella MA. Acute kidney injury in patients with cancer. N Engl J Med 2017;376:1770-1781.

[3] Salahudeen AK, Doshi SM, Pawar T, Nowshad G, Lahoti A, Shah P. Incidence rate, clinical correlates, and outcomes of AKI in patients admitted to a comprehensive cancer center. Clin J Am Soc Nephrol 2013;8:347-354.

[4] Christiansen CF, Johansen MB, Langeberg WJ, Fryzek JP, Sørensen HT. Incidence of acute kidney injury in cancer patients: a Danish population-based cohort study. Eur J Intern Med 2011;22:399-406.

[5] Jin J, Wang Y, Shen Q, Gong J, Zhao L, He Q. Acute kidney injury in cancer patients: a nationwide survey in China. Sci Rep 2019;9:3540.

[6] Darmon M, Vincent F, Canet E, et al. Acute kidney injury in critically ill patients with haematological malignancies: results of a multicentre cohort study from the Groupe de Recherche en Réanimation Respiratoire en Onco-Hématologie. Nephrol Dial Transplant 2015;30:2006-2013.

[7] Lahoti A, Kantarjian H, Salahudeen AK, et al. Predictors and outcome of acute kidney injury in patients with acute myelogenous leukemia or high-risk myelodysplastic syndrome. Cancer 2010;116:4063-4068.

[8] Darmon M, Ciroldi M, Thiery G, Schlemmer B, Azoulay E. Clinical review: specific aspects of acute renal failure in cancer patients. Crit Care 2006;10:211.

[9] Kitchlu A, McArthur E, Amir E, et al. Acute kidney injury in patients receiving systemic treatment for cancer: a population-based cohort study. J Natl Cancer Inst 2018 Nov 13 [Epub]. Doi: 10.1093/jnci/djy167.

[10] Canet E, Zafrani L, Lambert J, et al. Acute kidney injury in patients with newly diagnosed high-grade hematological malignancies: impact on remission and survival. PLoS One 2013;8:e55870.

[11] Soares M, Salluh JI, Carvalho MS, Darmon M, Rocco JR, Spector N. Prognosis of critically ill patients with cancer and acute renal dysfunction. J Clin Oncol 2006;24:40034010.

[12] Samuels J, Ng CS, Nates J, et al. Small increases in serum creatinine are associated with prolonged ICU stay and increased hospital mortality in critically ill patients with cancer. Support Care Cancer 2011;19:1527-1532. 
[13] Maccariello E, Valente C, Nogueira L, et al. Outcomes of cancer and non-cancer patients with acute kidney injury and need of renal replacement therapy admitted to general intensive care units. Nephrol Dial Transplant 2011;26: 537-543.

[14] Park MR, Jeon K, Song JU, et al. Outcomes in critically ill patients with hematologic malignancies who received renal replacement therapy for acute kidney injury in an intensive care unit. J Crit Care 2011;26:107.e1-107.e6.

[15] Libório AB, Abreu KL, Silva GB Jr, et al. Predicting hospital mortality in critically ill cancer patients according to acute kidney injury severity. Oncology 2011;80:160-166.

[16] Sweiss K, Calip GS, Oh AL, Rondelli D, Patel PR. Renal dysfunction within 90 days of FluBu4 predicts early and late mortality. Bone Marrow Transplant 2018;54:980-986.

[17] Darmon M, Thiery G, Ciroldi M, et al. Intensive care in patients with newly diagnosed malignancies and a need for cancer chemotherapy. Crit Care Med 2005;33:2488-2493.

[18] Canet E, Vincent F, Darmon M, Soares M. Acute kidney injury in hematological patients. Curr Opin Crit Care 2015; 21:549-558.

[19] Lundberg WB, Cadman ED, Finch SC, Capizzi RL. Renal failure secondary to leukemic infiltration of the kidneys. Am J Med 1977;62:636-642.

[20] Richmond J, Sherman RS, Diamond HD, Craver LF. Renal lesions associated with malignant lymphomas. Am J Med 1962;32:184-207.

[21] Barcos M, Lane W, Gomez GA, et al. An autopsy study of 1206 acute and chronic leukemias (1958 to 1982). Cancer 1987;60:827-837.

[22] Cambier JF, Ronco P. Onco-nephrology: glomerular diseases with cancer. Clin J Am Soc Nephrol 2012;7:17011712.

[23] Mir MA, Delamore IW. Metabolic disorders in acute myeloid leukaemia. Br J Haematol 1978;40:79-92.

[24] Eleutherakis-Papaiakovou V, Bamias A, Gika D, et al.; Greek Myeloma Study Group. Renal failure in multiple myeloma: incidence, correlations, and prognostic significance. Leuk Lymphoma 2007;48:337-341.

[25] Bladé J, Fernández-Llama P, Bosch F, et al. Renal failure in multiple myeloma: presenting features and predictors of outcome in 94 patients from a single institution. Arch Intern Med 1998;158:1889-1893.

[26] Rajkumar SV, Dimopoulos MA, Palumbo A, et al. International Myeloma Working Group updated criteria for the diagnosis of multiple myeloma. Lancet Oncol 2014;15: e538-e548.

[27] Burke JR Jr, Flis R, Lasker N, Simenhoff M. Malignant lymphoma with "myeloma kidney" acute renal failure. Am J Med 1976;60:1055-1060.

[28] Sanders PW, Booker BB. Pathobiology of cast nephropathy from human Bence Jones proteins. J Clin Invest 1992;89: 630-639.

[29] Basnayake K, Ying WZ, Wang PX, Sanders PW. Immunoglobulin light chains activate tubular epithelial cells through redox signaling. J Am Soc Nephrol 2010;21:11651173.

[30] Huang ZQ, Sanders PW. Localization of a single binding site for immunoglobulin light chains on human TammHorsfall glycoprotein. J Clin Invest 1997;99:732-736.

[31] Sanders PW. Pathogenesis and treatment of myeloma kidney. J Lab Clin Med 1994;124:484-488.

[32] Leung N, Gertz MA, Zeldenrust SR, et al. Improvement of cast nephropathy with plasma exchange depends on the diagnosis and on reduction of serum free light chains. Kidney Int 2008;73:1282-1288.

[33] Ying WZ, Wang PX, Sanders PW. Pivotal role of apoptosis signal-regulating kinase 1 in monoclonal free light chainmediated apoptosis. Am J Pathol 2012;180:41-47.

[34] Hutchison CA, Basnayake K, Cockwell P. Serum free light chain assessment in monoclonal gammopathy and kidney disease. Nat Rev Nephrol 2009;5:621-628.

[35] Dimopoulos MA, Sonneveld P, Leung N, et al. International Myeloma Working Group recommendations for the diagnosis and management of myeloma-related renal impairment. J Clin Oncol 2016;34:1544-1557.

[36] Nasr SH, Valeri AM, Sethi S, et al. Clinicopathologic correlations in multiple myeloma: a case series of 190 patients with kidney biopsies. Am J Kidney Dis 2012;59:786-794.

[37] Leung N, Gertz M, Kyle RA, et al. Urinary albumin excretion patterns of patients with cast nephropathy and other monoclonal gammopathy-related kidney diseases. Clin J Am Soc Nephrol 2012;7:1964-1968.

[38] Chanan-Khan AA, Kaufman JL, Mehta J, et al. Activity and safety of bortezomib in multiple myeloma patients with advanced renal failure: a multicenter retrospective study. Blood 2007;109:2604-2606.

[39] Jagannath S, Barlogie B, Berenson JR, et al.; SUMMIT/ CREST Investigators. Bortezomib in recurrent and/or refractory multiple myeloma. Initial clinical experience in patients with impared renal function. Cancer 2005;103: 1195-1200. 
[40] Scheid C, Sonneveld P, Schmidt-Wolf IG, et al. Bortezomib before and after autologous stem cell transplantation overcomes the negative prognostic impact of renal impairment in newly diagnosed multiple myeloma: a subgroup analysis from the HOVON-65/GMMG-HD4 trial. Haematologica 2014;99:148-154.

[41] Dimopoulos MA, Roussou M, Gavriatopoulou M, et al. Bortezomib-based triplets are associated with a high probability of dialysis independence and rapid renal recovery in newly diagnosed myeloma patients with severe renal failure or those requiring dialysis. Am J Hematol 2016;91:499-502.

[42] Dimopoulos MA, Delimpasi S, Katodritou E, et al. Significant improvement in the survival of patients with multiple myeloma presenting with severe renal impairment after the introduction of novel agents. Ann Oncol 2014;25:195200.

[43] Pönisch W, Holzvogt B, Plötze M, et al. Bendamustine and prednisone in combination with bortezomib (BPV) in the treatment of patients with newly diagnosed/untreated multiple myeloma. J Cancer Res Clin Oncol 2014;140: 1947-1956.

[44] Clark WF, Stewart AK, Rock GA, et al.; Canadian Apheresis Group. Plasma exchange when myeloma presents as acute renal failure: a randomized, controlled trial. Ann Intern Med 2005;143:777-784.

[45] Hutchison CA, Harding S, Mead G, et al. Serum free-light chain removal by high cutoff hemodialysis: optimizing removal and supportive care. Artif Organs 2008;32:910-917.

[46] Bridoux F, Carron PL, Pegourie B, et al.; MYRE Study Group. Effect of high-cutoff hemodialysis vs conventional hemodialysis on hemodialysis independence among patients with myeloma cast nephropathy: a randomized clinical trial. JAMA 2017;318:2099-2110.

[47] Finkel K, Fabbrini P. High cut-off hemodialysis for myeloma cast nephropathy - do we finally have an answer? J Onco-Nephrol 2017;1:67-70.

[48] Hingorani SR, Guthrie K, Batchelder A, et al. Acute renal failure after myeloablative hematopoietic cell transplant: incidence and risk factors. Kidney Int 2005;67:272-277.

[49] Parikh CR, Coca SG. Acute renal failure in hematopoietic cell transplantation. Kidney Int 2006;69:430-435.

[50] Gruss E, Bernis C, Tomas JF, et al. Acute renal failure in patients following bone marrow transplantation: prevalence, risk factors and outcome. Am J Nephrol 1995;15:473-479.

[51] Merouani A, Shpall EJ, Jones RB, Archer PG, Schrier RW.
Renal function in high dose chemotherapy and autologous hematopoietic cell support treatment for breast cancer. Kidney Int 1996;50:1026-1031.

[52] Parikh CR, Sandmaier BM, Storb RF, et al. Acute renal failure after nonmyeloablative hematopoietic cell transplantation. J Am Soc Nephrol 2004;15:1868-1876.

[53] Parikh CR, Yarlagadda SG, Storer B, et al. Impact of acute kidney injury on long-term mortality after nonmyeloablative hematopoietic cell transplantation. Biol Blood Marrow Transplant 2008;14:309-315.

[54] Kersting S, Koomans HA, Hené RJ, Verdonck LF. Acute renal failure after allogeneic myeloablative stem cell transplantation: retrospective analysis of incidence, risk factors and survival. Bone Marrow Transplant 2007;39:359-365.

[55] Go AS, Chertow GM, Fan D, McCulloch CE, Hsu CY. Chronic kidney disease and the risks of death, cardiovascular events, and hospitalization. N Engl J Med 2004;351: 1296-1305.

[56] Shingai N, Morito T, Najima Y, et al. Early-onset acute kidney injury is a poor prognostic sign for allogeneic SCT recipients. Bone Marrow Transplant 2015;50:1557-1562.

[57] Hingorani S. Renal complications of hematopoietic-cell transplantation. N Engl J Med 2016;374:2256-2267.

[58] Coppell JA, Richardson PG, Soiffer R, et al. Hepatic venoocclusive disease following stem cell transplantation: incidence, clinical course, and outcome. Biol Blood Marrow Transplant 2010;16:157-168.

[59] Bearman SI. The syndrome of hepatic veno-occlusive disease after marrow transplantation. Blood 1995;85:30053020.

[60] Attal M, Huguet F, Rubie H, et al. Prevention of hepatic veno-occlusive disease after bone marrow transplantation by continuous infusion of low-dose heparin: a prospective, randomized trial. Blood 1992;79:2834-2840.

[61] Gluckman E, Jolivet I, Scrobohaci ML, et al. Use of prostaglandin E1 for prevention of liver veno-occlusive disease in leukaemic patients treated by allogeneic bone marrow transplantation. Br J Haematol 1990;74:277-281.

[62] Bianco JA, Appelbaum FR, Nemunaitis J, et al. Phase I-II trial of pentoxifylline for the prevention of transplantrelated toxicities following bone marrow transplantation. Blood 1991;78:1205-1211.

[63] Richardson PG, Murakami C, Jin Z, et al. Multi-institutional use of defibrotide in 88 patients after stem cell transplantation with severe veno-occlusive disease and multisystem organ failure: response without significant 
toxicity in a high-risk population and factors predictive of outcome. Blood 2002;100:4337-4343.

[64] Chopra R, Eaton JD, Grassi A, et al. Defibrotide for the treatment of hepatic veno-occlusive disease: results of the European compassionate-use study. Br J Haematol 2000; 111:1122-1129.

[65] Laskin BL, Goebel J, Davies SM, Jodele S. Small vessels, big trouble in the kidneys and beyond: hematopoietic stem cell transplantation-associated thrombotic microangiopathy. Blood 2011;118:1452-1462.

[66] Laskin BL, Goebel J, Davies SM, et al. Early clinical indicators of transplant-associated thrombotic microangiopathy in pediatric neuroblastoma patients undergoing auto-SCT. Bone Marrow Transplant 2011;46:682-689.

[67] Batts ED, Lazarus HM. Diagnosis and treatment of transplantation-associated thrombotic microangiopathy: real progress or are we still waiting? Bone Marrow Transplant 2007;40:709-719.

[68] Siami K, Kojouri K, Swisher KK, Selby GB, George JN, Laszik ZG. Thrombotic microangiopathy after allogeneic hematopoietic stem cell transplantation: an autopsy study. Transplantation 2008;85:22-28.

[69] Kanamori H, Maruta A, Sasaki S, et al. Diagnostic value of hemostatic parameters in bone marrow transplantassociated thrombotic microangiopathy. Bone Marrow Transplant 1998;21:705-709.

[70] Matsuda Y, Hara J, Osugi Y, et al. Serum levels of soluble adhesion molecules in stem cell transplantation-related complications. Bone Marrow Transplant 2001;27:977-982.

[71] Ho VT, Cutler C, Carter S, et al. Blood and marrow transplant clinical trials network toxicity committee consensus summary: thrombotic microangiopathy after hematopoietic stem cell transplantation. Biol Blood Marrow Transplant 2005;11:571-575.

[72] Cho BS, Yahng SA, Lee SE, et al. Validation of recently proposed consensus criteria for thrombotic microangiopathy after allogeneic hematopoietic stem-cell transplantation. Transplantation 2010;90:918-926.

[73] Choi CM, Schmaier AH, Snell MR, Lazarus HM. Thrombotic microangiopathy in haematopoietic stem cell transplantation: diagnosis and treatment. Drugs 2009;69:183198.

[74] Kennedy GA, Kearey N, Bleakley S, Butler J, Mudie K, Durrant S. Transplantation-associated thrombotic microangiopathy: effect of concomitant GVHD on efficacy of therapeutic plasma exchange. Bone Marrow Transplant
2010;45:699-704.

[75] Cutler C, Henry NL, Magee C, et al. Sirolimus and thrombotic microangiopathy after allogeneic hematopoietic stem cell transplantation. Biol Blood Marrow Transplant 2005;11:551-557.

[76] Hahn T, Alam AR, Lawrence D, et al. Thrombotic microangiopathy after allogeneic blood and marrow transplantation is associated with dose-intensive myeloablative conditioning regimens, unrelated donor, and methylprednisolone T-cell depletion. Transplantation 2004;78:15151522.

[77] Uderzo C, Bonanomi S, Busca A, et al. Risk factors and severe outcome in thrombotic microangiopathy after allogeneic hematopoietic stem cell transplantation. Transplantation 2006;82:638-644.

[78] Worel N, Greinix HT, Leitner G, et al. ABO-incompatible allogeneic hematopoietic stem cell transplantation following reduced-intensity conditioning: close association with transplant-associated microangiopathy. Transfus Apher Sci 2007;36:297-304.

[79] Willems E, Baron F, Seidel L, Frère P, Fillet G, Beguin Y. Comparison of thrombotic microangiopathy after allogeneic hematopoietic cell transplantation with high-dose or nonmyeloablative conditioning. Bone Marrow Transplant 2010;45:689-693.

[80] Cho BS, Min CK, Eom KS, et al. Clinical impact of thrombotic microangiopathy on the outcome of patients with acute graft-versus-host disease after allogeneic hematopoietic stem cell transplantation. Bone Marrow Transplant 2008;41:813-820.

[81] Oran B, Donato M, Aleman A, et al. Transplant-associated microangiopathy in patients receiving tacrolimus following allogeneic stem cell transplantation: risk factors and response to treatment. Biol Blood Marrow Transplant 2007;13:469-477.

[82] Jodele S, Bleesing JJ, Mehta PA, et al. Successful early intervention for hyperacute transplant-associated thrombotic microangiopathy following pediatric hematopoietic stem cell transplantation. Pediatr Transplant 2012;16:E39E42.

[83] Marr H, McDonald EJ, Merriman E, et al. Successful treatment of transplant-associated microangiopathy with rituximab. NZ Med J 2009;122:72-74.

[84] Naina HV, Gertz MA, Elliott MA. Thrombotic microangiopathy during peripheral blood stem cell mobilization. $J$ Clin Apher 2009;24:259-261. 
[85] Carella AM, D'Arena G, Greco MM, Nobile M, Cascavilla N. Rituximab for allo-SCT-associated thrombotic thrombocytopenic purpura. Bone Marrow Transplant 2008;41:10631065.

[86] Au WY, Ma ES, Lee TL, et al. Successful treatment of thrombotic microangiopathy after haematopoietic stem cell transplantation with rituximab. Br J Haematol 2007; 137:475-478.

[87] Lopes JA, Jorge S. Acute kidney injury following HCT: incidence, risk factors and outcome. Bone Marrow Transplant 2011;46:1399-1408.

[88] Kogon A, Hingorani S. Acute kidney injury in hematopoietic cell transplantation. Semin Nephrol 2010;30:615-626.

[89] Mirrakhimov AE, Voore P, Khan M, Ali AM. Tumor lysis syndrome: a clinical review. World J Crit Care Med 2015;4: 130-138.

[90] Wilson FP, Berns JS. Tumor lysis syndrome: new challenges and recent advances. Adv Chronic Kidney Dis 2014;21: 18-26.

[91] Cairo MS, Coiffier B, Reiter A, Younes A; TLS Expert Panel. Recommendations for the evaluation of risk and prophylaxis of tumour lysis syndrome (TLS) in adults and children with malignant diseases: an expert TLS panel consensus. Br J Haematol 2010;149:578-586.

[92] Durani U, Shah ND, Go RS. In-hospital outcomes of tumor lysis syndrome: a population-based study using the national inpatient sample. Oncologist 2017;22:1506-1509.

[93] Cairo MS, Bishop M. Tumour lysis syndrome: new therapeutic strategies and classification. Br J Haematol 2004; 127:3-11.

[94] Shimada M, Johnson RJ, May WS Jr, et al. A novel role for uric acid in acute kidney injury associated with tumour lysis syndrome. Nephrol Dial Transplant 2009;24:29602964.

[95] Spina M, Nagy Z, Ribera JM, et al.; FLORENCE Study Group. FLORENCE: a randomized, double-blind, phase III pivotal study of febuxostat versus allopurinol for the prevention of tumor lysis syndrome (TLS) in patients with hematologic malignancies at intermediate to high TLS risk. Ann Oncol 2015;26:2155-2161.

[96] Cortes J, Moore JO, Maziarz RT, et al. Control of plasma uric acid in adults at risk for tumor Lysis syndrome: efficacy and safety of rasburicase alone and rasburicase followed by allopurinol compared with allopurinol alone--results of a multicenter phase III study. J Clin Oncol 2010;28:42074213.
[97] Vadhan-Raj S, Fayad LE, Fanale MA, et al. A randomized trial of a single-dose rasburicase versus five-daily doses in patients at risk for tumor lysis syndrome. Ann Oncol 2012; 23:1640-1645.

[98] Sherwood GB, Paschal RD, Adamski J. Rasburicase-induced methemoglobinemia: case report, literature review, and proposed treatment algorithm. Clin Case Rep 2016;4: 315-319.

[99] Rosner MH, Dalkin AC. Onco-nephrology: the pathophysiology and treatment of malignancy-associated hypercalcemia. Clin J Am Soc Nephrol 2012;7:1722-1729.

[100] Mazzone PJ, Arroliga AC. Endocrine paraneoplastic syndromes in lung cancer. Curr Opin Pulm Med 2003;9:313320.

[101] Pargger H, Kaufmann MA, Drop LJ. Renal vascular hyperresponsiveness to elevated ionized calcium in spontaneously hypertensive rat kidneys. Intensive Care Med 1998; 24:61-70.

[102] LeGrand SB, Leskuski D, Zama I. Narrative review: furosemide for hypercalcemia: an unproven yet common practice. Ann Intern Med 2008;149:259-263.

[103] Dietzek A, Connelly K, Cotugno M, Bartel S, McDonnell AM. Denosumab in hypercalcemia of malignancy: a case series. J Oncol Pharm Pract 2015;21:143-147.

[104] Adhikaree J, Newby Y, Sundar S. RE: denosumab for patients with persistent or relapsed hypercalcemia of malignancy despite recent bisphosphonate treatment. J Natl Cancer Inst 2015;107:dju509.

[105] Cardella CJ, Birkin BL, Rapoport A. Role of dialysis in the treatment of severe hypercalcemia: report of two cases successfully treated with hemodialysis and review of the literature. Clin Nephrol 1979;12:285-290.

[106] Perazella MA. Onco-nephrology: renal toxicities of chemotherapeutic agents. Clin J Am Soc Nephrol 2012;7:17131721.

[107] Shirali AC, Perazella MA. Tubulointerstitial injury associated with chemotherapeutic agents. Adv Chronic Kidney Dis 2014;21:56-63.

[108] Porta C, Cosmai L, Gallieni M, Pedrazzoli P, Malberti F. Renal effects of targeted anticancer therapies. Nat Rev Nephrol 2015;11:354-370.

[109] Perazella MA, Moeckel GW. Nephrotoxicity from chemotherapeutic agents: clinical manifestations, pathobiology, and prevention/therapy. Semin Nephrol 2010;30:570-581.

[110] Perazella MA, Izzedine H. New drug toxicities in the onconephrology world. Kidney Int 2015;87:909-917. 
[111] Teuma C, Perier-Muzet M, Pelletier S, et al. New insights into renal toxicity of the B-RAF inhibitor, vemurafenib, in patients with metastatic melanoma. Cancer Chemother Pharmacol 2016;78:419-426.

[112] Izzedine H, El-Fekih RK, Perazella MA. The renal effects of ALK inhibitors. Invest New Drugs 2016;34:643-649.

[113] Cortazar FB, Marrone KA, Troxell ML, et al. Clinicopathological features of acute kidney injury associated with immune checkpoint inhibitors. Kidney Int 2016;90:638-647.

[114] Shirali AC, Perazella MA, Gettinger S. Association of acute interstitial nephritis with programmed cell death 1 inhibitor therapy in lung cancer patients. Am J Kidney Dis 2016; 68:287-291.

[115] Izzedine H, Perazella MA. Thrombotic microangiopathy, cancer, and cancer drugs. Am J Kidney Dis 2015;66:857868.

[116] Glezerman I, Kris MG, Miller V, Seshan S, Flombaum CD. Gemcitabine nephrotoxicity and hemolytic uremic syndrome: report of 29 cases from a single institution. Clin Nephrol 2009;71:130-139.

[117] Markowitz GS, Bomback AS, Perazella MA. Drug-induced glomerular disease: direct cellular injury. Clin J Am Soc Nephrol 2015;10:1291-1299.

[118] Perazella MA, Markowitz GS. Bisphosphonate nephrotoxicity. Kidney Int 2008;74:1385-1393.

[119] Markowitz GS, Appel GB, Fine PL, et al. Collapsing focal segmental glomerulosclerosis following treatment with high-dose pamidronate. J Am Soc Nephrol 2001;12:11641172.

[120] de Miguel D, García-Suárez J, Martín Y, Gil-Fernández JJ, Burgaleta C. Severe acute renal failure following highdose methotrexate therapy in adults with haematological malignancies: a significant number result from unrecognized co-administration of several drugs. Nephrol Dial Transplant 2008;23:3762-3766.

[121] Wall SM, Johansen MJ, Molony DA, DuBose TD Jr, Jaffe N, Madden T. Effective clearance of methotrexate using highflux hemodialysis membranes. Am J Kidney Dis 1996;28:
846-854.

[122] Widemann BC, Schwartz S, Jayaprakash N, et al. Efficacy of glucarpidase (carboxypeptidase g2) in patients with acute kidney injury after high-dose methotrexate therapy. Pharmacotherapy 2014;34:427-439.

[123] Sahni V, Choudhury D, Ahmed Z. Chemotherapy-associated renal dysfunction. Nat Rev Nephrol 2009;5:450-462.

[124] Gurevich F, Perazella MA. Renal effects of anti-angiogenesis therapy: update for the internist. Am J Med 2009;122: 322-328.

[125] Izzedine H, Mangier M, Ory V, et al. Expression patterns of RelA and c-mip are associated with different glomerular diseases following anti-VEGF therapy. Kidney Int 2014;85: 457-470.

[126] Jhaveri KD, Sakhiya V, Fishbane S. Nephrotoxicity of the BRAF inhibitors vemurafenib and dabrafenib. JAMA Oncol 2015;1:1133-1134.

[127] Perazella MA, Izzedine H. Crizotinib: renal safety evaluation. JOnco-Nephrol 2017;1:49-56.

[128] Markowitz GS, Nasr SH, Stokes MB, D'Agati VD. Treatment with IFN-\{alpha\}, -\{beta\}, or -\{gamma\} is associated with collapsing focal segmental glomerulosclerosis. Clin J Am Soc Nephrol 2010;5:607-615.

[129] Schwartz RN, Stover L, Dutcher JP. Managing toxicities of high-dose interleukin-2. Oncology (Williston Park) 2002; 16(11 Suppl 13):11-20.

[130] Postow MA, Callahan MK, Wolchok JD. Immune checkpoint blockade in cancer therapy. J Clin Oncol 2015;33: 1974-1982.

[131] Gill S, Maus MV, Porter DL. Chimeric antigen receptor T cell therapy: 25years in the making. Blood Rev 2016;30: 157-167.

[132] Bonifant CL, Jackson HJ, Brentjens RJ, Curran KJ. Toxicity and management in CAR T-cell therapy. Mol Ther Oncolytics 2016;3:16011.

[133] Brudno JN, Kochenderfer JN. Toxicities of chimeric antigen receptor T cells: recognition and management. Blood 2016;127:3321-3330. 